\title{
EVOLUCIÓN FUTURA DE LOS CICLONES CON CARACTERÍSTICAS TROPICALES EN LA CUENCA MEDITERRÁNEA: EFECTOS DE UTILIZAR MODELOS DE ALTA RESOLUCIÓN O CON ACOPLAMIENTO ATMÓSFERA-OCÉANO
}

\author{
Jesús Gutiérrez-Fernández ${ }^{(1)}$, Juan Jesús González-Alemán ${ }^{(1)}$ \\ y Miguel Ángel Gaertner ${ }^{(1,2)}$ \\ (1) Instituto de Ciencias Ambientales (ICAM). Universidad de Castilla-La Mancha, \\ (jesus.gutierrez1@alu.uclm.es) (juanjesus.gonzalez@uclm.es) \\ (2) Facultad de Ciencias del Medio Ambiente y Bioquímica. Universidad de Castilla-La Mancha. \\ (miguel.gaertner@uclm.es)
}

\section{Introducción}

Los ciclones con características tropicales son poco frecuentes en la cuenca mediterránea debido a que las condiciones atmosféricas y oceánicas son poco favorables para su desarrollo y mantenimiento. En el mar Mediterráneo, este tipo de ciclones son llamados medicanes ("Mediterranean Hurricanes"). En los últimos años, se han observado medicanes con una fuerte intensidad como el ocurrido en noviembre de 2011 (Miglietta et al., 2013).

Los medicanes presentan características tropicales como son la presencia de núcleo cálido, simetría e intensa convección. Estos ciclones están asociados a fenómenos extremos como son fuertes vientos, precipitaciones torrenciales y fuerte oleaje, afectando sobre todo a zonas costeras.

Este tipo de ciclones tienen origen en bajas presiones extratropicales que llevan asociadas una potente Vaguada. Esta vaguada da lugar a una baja presión secundaría que al pasar a zonas del Mar Mediterráneo pierde sus características baroclínicas y sufre un proceso de transición tropical.

Para que los procesos de transición tropical tengan lugar es necesario que la cizalla del viento en altura sea débil (fig. 1), si esto ocurre el núcleo de la baja en altura y en superficie quedan alienados, lo que favorece fuerte convección, que además da lugar a la liberación
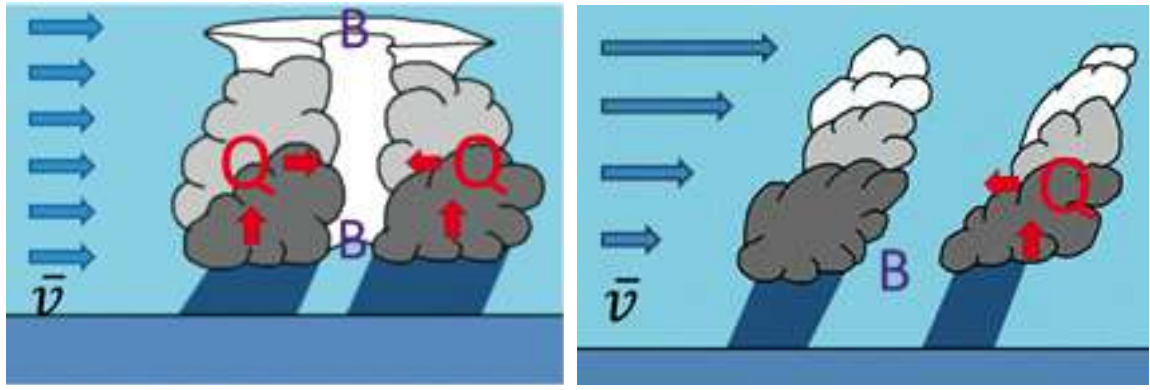

Fig. 1- Representación de las condiciones de cizalla del viento en altura que permiten el desarrollo de medicanes (fig. 1a) y las que no son favorables (fig. 1b). 
de calor latente en el centro del ciclón lo que propicia la formación de su núcleo cálido. Tras esto el ciclón comienza a perder la estructura frontal y su estructura asimétrica.

Si la cizalla del viento es intensa, las bajas en altura y en superficie se encontrarían desfasadas, dando como resultado que la convección sea menos intensa y no se produzca desarrollo de medicane.

\section{Material y métodos}

Los datos utilizados en el análisis son datos proporcionados por simulaciones de modelos regionales de clima, para escenarios de clima futuro RCP85 hasta 2100, utilizando

\begin{tabular}{|c|c|c|}
\hline MODELO ACM & $\begin{array}{c}\text { Acoplamiento (Oceano- } \\
\text { Tierra). }\end{array}$ & Resolución horizontal. \\
\hline \multirow{3}{*}{ AWI-REMO } & Acoplado (C) & $50 \mathrm{~km}$ (LR) \\
& No acoplado (U) & $50 \mathrm{~km}$ (LR) \\
\hline & Acoplado (C) & $25 \mathrm{~km}$ (HR) \\
\hline \multirow{3}{*}{ CNRM-ALADIN52 } & No acoplado (U) & $25 \mathrm{~km}$ (HR) \\
\hline CSC-REMO & No acoplado (U) & $50 \mathrm{~km}$ (LR) \\
\hline SMHI-RCA4 & No acoplado (U) & $12,5 \mathrm{~km}$ (HR) \\
\hline MODELO GLOBAL & No acoplado (U) & $50 \mathrm{~km}$ (LR) \\
\hline GFDL & No acoplado (U) & $50 \mathrm{~km}$ (LR) \\
\hline
\end{tabular}

El análisis de detección de medicanes incluye el método de Picornell, (Picornell et al., 2001) para la detección de ciclones, adaptado a la detección de ciclones mesoescalares, y el empleo del método de Hart (Hart, 2003) para detectar ciclones que presenten estructura y características tropicales.

En este método se utilizan tres variables fundamentales para caracterizar la estructura térmica de cualquier ciclón. El parámetro de simetría (B), nos permite dar una idea de si el sistema presenta características frontales o no. Para este estudio se determina que debe de ser mayor de cero $(B>0)$. Tras esto se mira también el viento térmico en la alta $\left(V_{-} \mathrm{T}^{\wedge} \mathrm{U}\right) \mathrm{y}$ baja $\left(V_{-} T^{\wedge} L\right)$ troposfera, esto nos da una idea de la estructura térmica vertical en el centro del ciclón. En este caso estos valores tienen que ser superiores a cero para identificar al ciclón. diferentes resoluciones horizontales así como modelos acoplados y no acoplados. (Tabla 1). Las simulaciones fueron hechas en el marco de trabajo de los programas europeos MedCORDEX y EuroCORDEX.

Tabla. 1.- Modelos utilizados para realizar este trabajo.

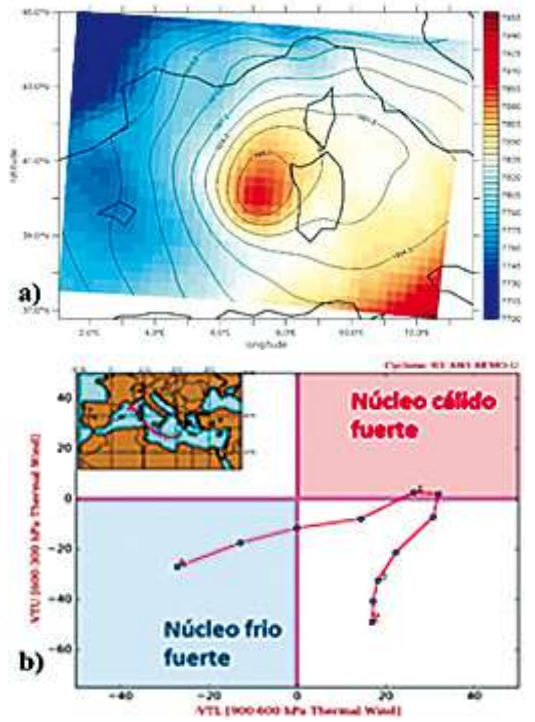

Fig. 2- La subfigura a muestra un mapa de diferencia de geopotencial (900-300 hPa) en sombreado y en contornos la presión en hPa. Mientras que la subfigura b corresponde con los diagramas de fase de vida de un ciclón simulado con AWI-REMO-C-HR. 
Por último se aplica un umbral de velocidad máxima del viento en el ciclón de $16,5 \mathrm{~m} / \mathrm{s}$ en el caso de modelos de baja resolución horizontal y de $17,5 \mathrm{~m} / \mathrm{s}$ en modelos de alta resolución horizontal.

\section{Resultados}

En este apartado se muestran los resultados conseguidos tras realizar un estudio estadístico de los medicanes obtenidos.

\subsection{Frecuencia mensual de medicanes}
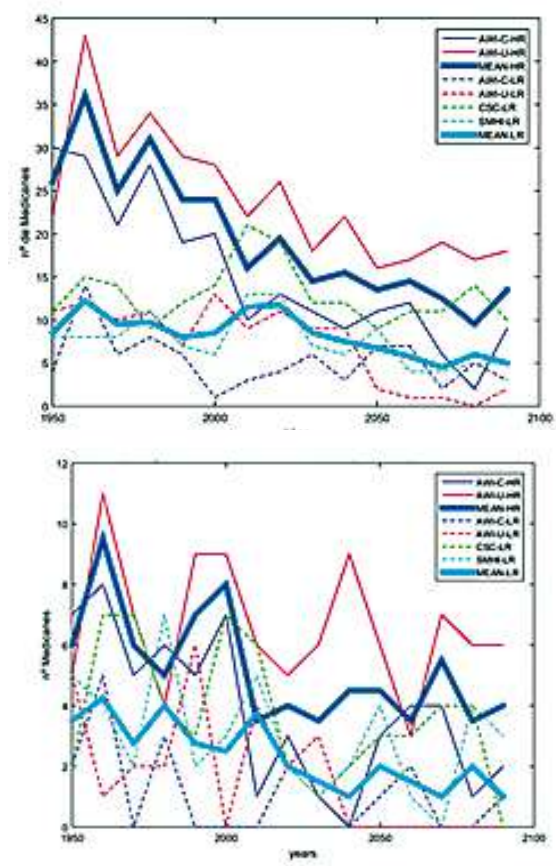

Fig. 3- Frecuencia decadal de medicanes para modelos de alta y baja Resolución desde 19502100 .

En la fig. 3 se puede ver que la frecuencia decadal de medicanes es superior en los modelos de alta resolución debido a que estos presentan una mayor sensibilidad a detectar este tipo de fenómenos de mesoescala.

La tendencia de medicanes desde 19502100 muestra una inclinación a descender a finales de siglo, este descenso es más pronunciado en los modelos de alta resolución, donde la tendencia es significativa al 95\%.

Otra parte de este estudio decadal consistió en tomar los medicanes que presentan un ciclo de vida superior a 12 horas.

Fig. 4- Frecuencia decadal de medicanes para modelos de alta y baja resolución desde 19502100 para medicanes con un ciclo de vida igual o superior a 12 horas.

Los resultados obtenidos muestran que en modelos de HR se observa una tendencia a disminuir su frecuencia hasta principios del S.XXI, a partir de entonces se observa una tendencia a mantenerse $\mathrm{o}$ a aumentar de forma ligera la frecuencia de dichos ciclones.

\subsection{Frecuencia mensual de medicanes}

Los medicanes no presentan una homogeneidad de aparición a lo largo del año, esto da lugar a un ciclo mensual de medicanes que según se puede observar en la fig. 5 , presenta un máximo de frecuencia durante los meses de invierno (sobre todo en enero) mientras que durante los meses de verano se observa un mínimo estacionario de frecuencia. 


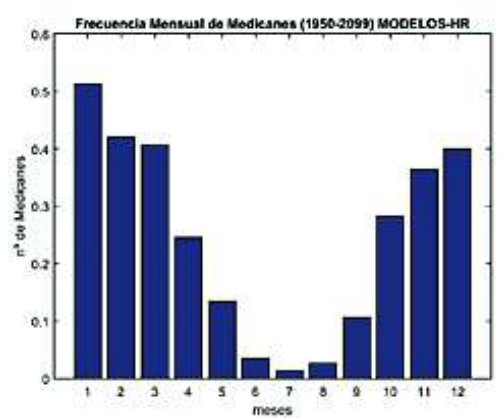

Fig. 6.- Frecuencia mensual de medicanes en modelos de alta y baja resolución para clima pasado y futuro.

A grandes rasgos, se puede ver que la frecuencia de medicanes desciende en todos los meses de cara a finales del S.XXI, pero este descenso es más pronunciado durante los meses de invierno. Haciendo un análisis global por estaciones, se confirma este descenso como se puede ver en la Tabla 2.

\begin{tabular}{|c|c|c|c|c|c|c|}
\hline & \multicolumn{2}{|c|}{ Modelos HR } & \multirow{2}{*}{ Variación HR } & \multicolumn{2}{|c|}{ Modelos LR } & \multirow{2}{*}{ Variación HR } \\
\hline Estaciones & $1950-2005$ & $2006-2100$ & & $1950-2005$ & $2006-2100$ & \\
\hline \begin{tabular}{l|l}
1 Imvierno \\
\end{tabular} & 1,77 & 1,02 & $-0,75$ & 0,75 & 0,39 & $-0,36$ \\
\hline 2 Primavera & 0,54 & 0,32 & $-0,22$ & 0,13 & 0,12 & $-0,02$ \\
\hline 3 Verano & 0,19 & 0,12 & $-0,07$ & 0,08 & 0,06 & $-0,02$ \\
\hline \begin{tabular}{l|l}
4 & Otoño
\end{tabular} & 1,29 & 0,87 & $-0,42$ & 0,48 & 0,40 & $-0,08$ \\
\hline
\end{tabular}

Tabla. 2.- frecuencia de medicanes por año en cada una de las estaciones y el porcentaje de variación entre clima pasado y clima futuro.

A finales de siglo es posible que la frecuencia de aparición de medicanes durante invierno y otoño tienda a igualarse en vista a los resultados obtenidos.

\subsection{Intensidad de medicanes}

A la hora de medir la intensidad de estos ciclones se ha utilizado la variable de velocidad máxima del viento $(\mathrm{m} / \mathrm{s})$ en el ciclón. Los resultados obtenidos muestran que los modelos de alta resolución muestran una mayor intensidad de viento que los modelos simulados en baja resolución, como se puede ver en fig. 7 .

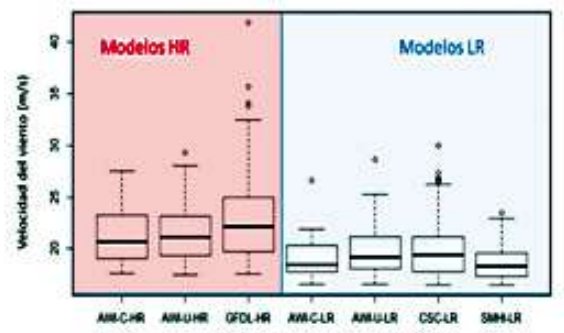

Fig. 7.- Boxplots de velocidad máxima del viento $(\mathrm{m} / \mathrm{s})$ para modelos de alta (HR) y baja (LR) resolución.

Al realizar el estudio de la velocidad máxima de viento por cincuentenas para los modelos de alta resolución (fig. 8), se observa que no hay un comportamiento común claro de la velocidad máxima del 
viento en los diferentes modelos. AWI-REMO-C y CNRM muestran un ligero descenso o mantenimiento de la intensidad de medicanes de cara a finales de este siglo, mientras que AWI-REMO-U y GFDL, muestran una tendencia a aumentar la intensidad de estos ciclones, de forma más acusada en el caso del GFDL.

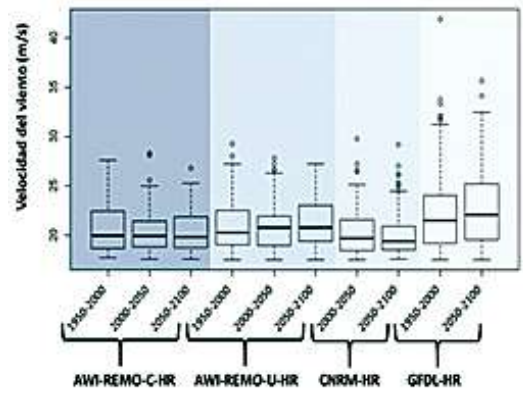

Fig. 8.- Boxplots de velocidad máxima del viento $(\mathrm{m} / \mathrm{s})$ para modelos de alta resolución horizontal, divididos en periodos de cincuenta años para estudiar su evolución futura.

Fig. 9.- Frecuencia decadal de medicanes para el modelo AWI-REMO de alta resolución

horizontal tanto para la simulación acoplada $(C)$ como para la simulación sin acoplar (U) desde 1950-2100.

Por tanto no se observa un patrón claro de comportamiento futuro de la intensidad de viento, sino que en este aspecto la incertidumbre es bastante elevada.

3.4 Diferencias entre los pares del Modelo AWIREMO con acoplamiento atmósfera-océano y sin acoplamiento

Uno de los principales objetivos de este trabajo es el estudio del efecto de utilizar modelos con acoplamiento atmósfera-océano y aquellos que no lo tienen (Gaertner et al., 2016). Para ello se ha utilizado el Modelo regional
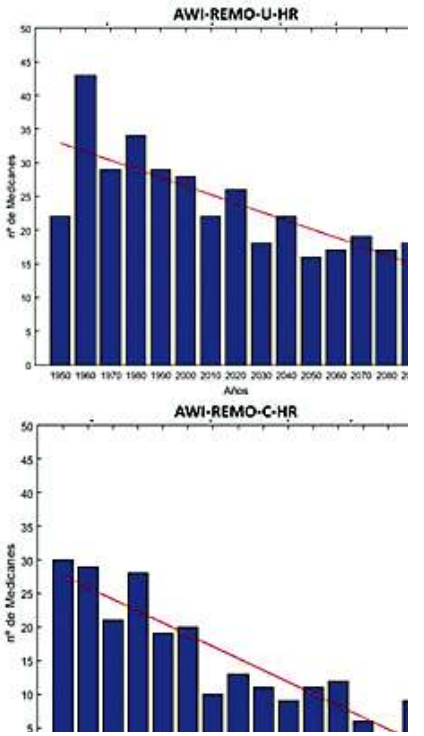
Climático AWI-REMO, el cual tiene los dos pares de

SHLREMO-L-HR
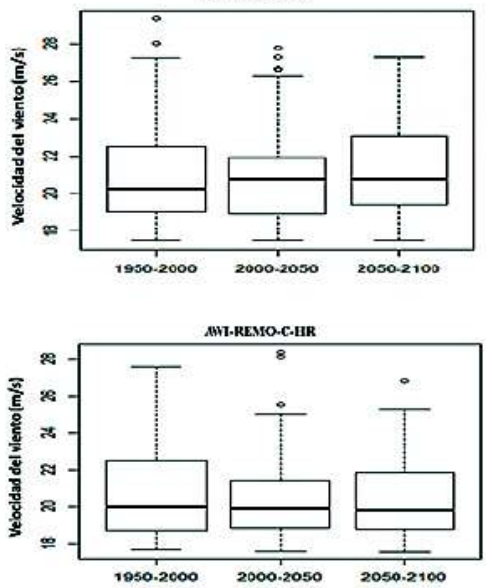
simulaciones.

Los resultados obtenidos muestran que hay diferencias entre ambas simulaciones, y que eso afecta a cuestiones anteriormente estudiadas como son la frecuencia decadal o la intensidad de los mismos.

En cuanto a la frecuencia década (fig. 9.) se observa que la disminución de medicanes de cara

Fig. 10.- Boxplots de velocidad máxima del viento $(\mathrm{m} / \mathrm{s})$ para el Modelo AWI-REMO de alta resolución horizontal tanto para la simulación acoplada $(C)$ como para la simulación sin acoplar (U), divididos en periodos de cincuenta años. 
a finales de siglo es más notable en la simulación acoplada (AWI-REMO-C-HR) que en la simulación no acoplada (AWI-REMO-U-HR), donde el descenso es menos acusado.

Por otro lado, el estudio de la intensidad máxima de viento para los dos pares de simulaciones, ofrece información diferente (fig. 10.) El modelo AWI-REMO-U-HR muestra un ligero aumento de la intensidad de los mismos mientras que la simulación acoplada (AWIREMO-C-HR) aprecia una ligera disminución de la intensidad de los mismos.

Es decir existen diferencias a simple vista de utilizar modelos acoplados a utilizar no acoplados.

\subsection{Ciclón simulado con AWI-REMO-U-HR (agosto de 1998)}

Durante el periodo de tratamiento de datos para determinar aquellos ciclones que pueden ser considerados como medicanes, apareció el caso de un ciclón en zonas del Este del Mediterráneo que era muy intenso el cual se generó en el mes de Agosto, un mes donde

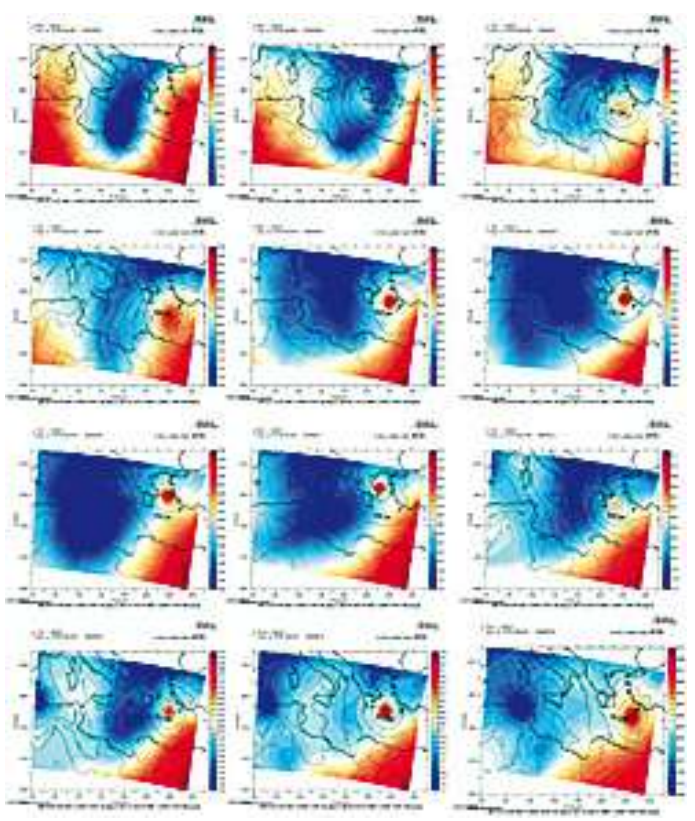
es difícil encontrar medicanes, como se comentó anteriormente en el apartado 3.2.

Fig. 11.- Mapas de diferencias de geopotencial $(900-300 \mathrm{hPa})$ en sombreado y en contornos la presión en hPa, que muestran la evolución del medicane detectado en ggosto de 1998 por el modelo AWI-REMO-U-HR en zonas del este del Mediterráneo.

El ciclón en cuestión (fig.11) duró 12 días (5 - 17 de Agosto), presentaba un núcleo cálido muy desarrollado, con una intensidad de viento que podía ser comparada con la de un Huracán de Categoría 1 o 2 y llegando a afectar a zonas de Grecia, Malta y Turquía.

Este medicane fue detectado por el modelo AWI-REMO-UHR, es decir la simulación histórica del modelo no acoplado de alta resolución. Sin embargo al comprobar si el modelo AWI-REMO-C-HR (simulación acoplado), había detectado dicho ciclón, esta simulación no lo había hecho.

Las posibles diferencias de detección se pueden encontrar en que el modelo AWI-REMO-U-HR haya potenciado de manera excesiva el ciclón sin tener en cuenta las condiciones que permiten la retroalimentación que permiten el mantenimiento y desarrollo del mismo.

Durante los meses de verano, la temperatura del mar es muy elevada (superior a los $25^{\circ} \mathrm{C}$ ), sin embargo la termoclina en esta época del año es muy somera, esto da lugar a 
que cuando se desarrolla una perturbación en zonas del Mediterráneo, la masa de agua superficial se vea reemplazada por agua más profunda y a más baja temperatura debido a la inestabilidad que genera la baja presión sobre el mar. Esto da lugar a que los flujos de calor latente no sean intensos y favorezcan los procesos de convección y mantenimiento de este tipo de ciclones con características tropicales.

Este efecto, sí que ha sido tomado en cuenta por el modelo AWI-REMO-C-HR, en este caso el acoplamiento atmosfera-océano, no permite que se favorezcan este tipo de ciclones durante los meses de verano en el periodo histórico.

\section{Conclusiones}

Emplear modelos climáticos de alta resolución horizontal (HR), ayuda a tener mayor sensibilidad e intentar detectar un número de medicanes más acorde con los datos observados que si utilizamos modelos de baja resolución. Estos modelos ven que hay una tendencia general a descender la frecuencia de medicanes en las próxima décadas, aun así se observa una gran variabilidad decadal, que deberá ser analizada en trabajos siguientes.

En cuanto a la intensidad, con los resultados obtenidos no se pueden sacar conclusiones absolutas, debido a que no todos los modelos muestran resultados iguales.

Uno de los grandes objetivos de este trabajo era también analizar las diferencias de utilizar modelos acoplados y no acoplados. Se puede decir que los modelos acoplados reproducen de manera más fiel a la realidad el clima pasado. Por tanto esto nos empuja a pensar que debido a que simulan mejor la relación entre las condiciones atmosféricas y oceánicas, es posible que en el futuro, el empleo de estos modelos nos permita un mejor análisis de estos ciclones.

\section{Referencias}

- Akhtar, N., Brauch, J., Dobler, A., Béranger, K., Ahrens, B., 2014. Medicanes in an oceanatmosphere coupled regional climate model. Nat. Hazards Earth Syst. Sci., 14, 2189-2201, 2014. doi:10.5194/nhess-14-2189-2014

- Carrío, D.S, Homar V., Jans, A., Romero R., Picornell, M.A., 2017. Tropicalizations process of the 7 November 2014 Mediterranean cyclone: Numerical sensitivity study. Atmospheric Research 197 (2017), 300-312.

- Cavicchia, L., Von Storch, H., Gualdi, S., 2013. A lomg-term climatology of medicanes. Clim Dyn 43:1183-1195. DOI 10.1007/s00382-013-1893-7.

- Gaertner, M.A., González-Alemán, J.J. et al., 2016. Simulation of medicanes over the Mediterranean Sea in a regional climate model ensemble: impact of ocean-atmosphere coupling and increased resolution. Climate Dynamics, http://dx.doi.org/10.1007/s00382-016-3456-1

- Hart, R. E., 2003. A cyclone phase space derived from thermal wind and termal asymmetry. Monthly Weather Review, 131(4), 585-616.

- Miglietta, M. M., Laviola, S., Malvaldi, A., Conte, D., Levizzani, V., \& Price, C., 2013. Analysis of tropical approach. Geophysical Research Letters, 40(10), 2400-2405.

- Picornell, M. A., Jansa, A., Genovés, A., \& Campins, J. (2001). Automated database of mesocyclones from the HIRLAM (INM) $\square 0.5^{\circ}$ analyses in the western Mediterranean. International Journal of Climatology, 21(3), 335-354. 